\section{Military Technical College Kobry El-Kobbah, Cairo, Egypt}

\author{
$8^{\text {th }}$ International Conference \\ on Electrical Engineering \\ ICEENG 2012
}

\title{
The generation of Bessel beams using nanoscale planar slit arrays
}

\author{
By \\ Ismail M. Nassar* Aladin H. Kamel** Diaa Khalil* Omar A. Omar*
}

\section{Abstract:}

In this paper we propose a nanoscale planar lens structure for generating Bessel beam like fields to achieve large focal lengths and depth of fields. The structure consists of sub wavelength slits of different widths constructed on a thin metallic slab that is surrounded by air on both sides. The proposed structure is simulated using a modal technique that represents the structure as a waveguide and solves for the modes of the waveguide. Although diffraction affects the behavior of the generated beam from that of conventional Bessel beams, the simulation results show that the proposed structure is very promising for obtaining a very compact Bessel beam generator.

\section{Keywords:}

Planar lens, Bessel beam, Modal technique

* Faculty of Engineering, Ain Shams University, Abbassia, Cairo, Egypt

** Advanced Industrial, Technical and Engineering Center, Cairo, Egypt 


\section{Introduction:}

Nanofabrication techniques have evolved recently enabling the realization of nanoscale structures that were previously not considered. These techniques include deep UV lithography, electron-beam lithography and focused ion beam milling. Investigating the behaviour of such nanoscale structures has therefore become a very active field of research in the recent years [1]-[2]-[3]. On the other hand, the development in Optical Micro-Electro-Mechanical MEMS systems required the propagation of low dimensional optical beams in the free space. Such beams suffer from diffraction and thus have limited working distances. One way to overcome this diffraction problem is to use low diffraction beams like the Bessel beam. The generation of such Bessel beams with a technology compatible to the nano/micro electronic technology is thus of great importance.

In this paper, we investigate the possibility of generating Bessel beams using an array of nanoscale planar slits. For simplicity, we assume a 1D structure to reduce the calculation time. -First, the diffraction effects on the behaviour of 1D Bessel beams is studied by reducing the aperture size of an ordinary axicon lens to sub-wavelength dimensions. We then investigate the replacement of an axicon lens by an array of nanoscale slits generating similar wavefronts to generate the required Bessel beams.

In our study we use a modal technique based on representing the slits forming the lens structure by waveguides with equivalent dimensions. Solving for the modes of these nanoscale waveguides, the output field from the lens is calculated. This modal technique shows significant advantage concerning simulation time when compared to other techniques, such as the finite difference time domain technique [4]-[5].

The paper is divided as follows: The proposed structure is illustrated in part 2. The waveguide modes technique used in the simulations is explained in part 3. In part 4, the simulation results are shown, while these results are discussed and a conclusion is given in part 5.

\section{The Proposed Structure:}

The proposed structure is shown in Figure 1. It consists of a thin metallic slab with a number of sub wavelength equally spaced slits. The widths of the slits are varied in order to synthesis the phase shift of the field output from the slit. By controlling the output phases from the different slits, the wavefront of the entire lens structure can be fitted to approach that of a Bessel beam originating from an axicon lens. In our case, however, we assume a 1D structure for simplicity and to reduce the required calculation time. Our 1D structure will be thus, corresponding to a prism. The metallic slab is surrounded with air on both sides and the slits are assumed to be all filled with air. In 
part 4, we investigate the reasonable ranges for the spacing between the slits and the overall size of the proposed micro structure.

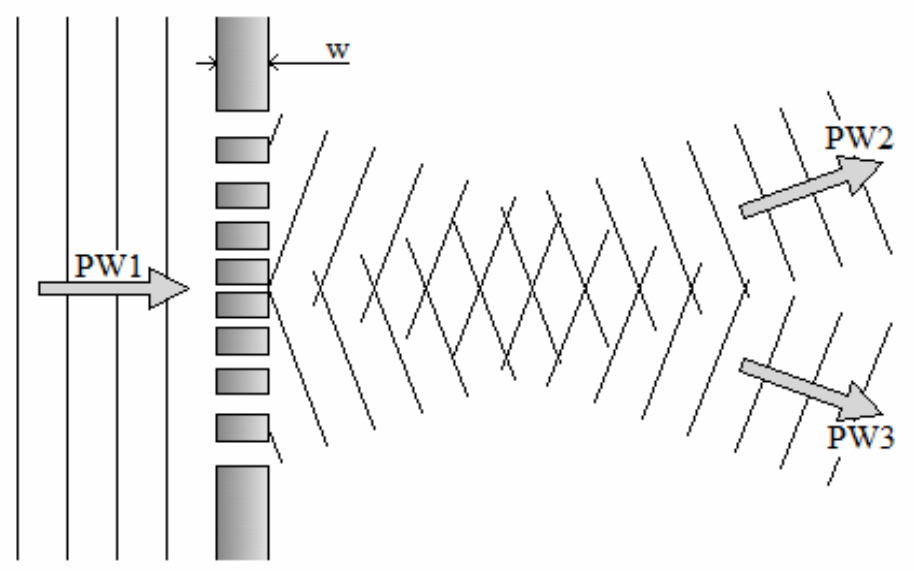

Figure (1): The proposed planar lens consisting of a set of 33 equally spaced nanoscale apertures of different widths in a conducting slab of thickness w=500nm. For illustration, only 9 apertures are shown.

\section{Waveguide Modes Technique:}

The technique used for studying the structure is based on representing the structure by a 1D waveguide with the appropriate values for the relative permittivity and the dimensions. A mode solver [6] is used to find the modes of the 1D structure which gives the transverse $E_{x}$ and $E_{y}$ field patterns together with the propagation constant $\beta$ along the z-direction for each mode. The mode solver is given a criterion to find the modes with the least magnitude for the imaginary part of the decay constant up to the $\mathrm{M}^{\text {th }}$ modes. These modes are the ones with the largest contribution to the output field.

It is assumed that only M modes are excited and propagate through the waveguide in the forward and backward directions each with specific amplitude. In order to determine these amplitudes an algebraic linear system is constructed. The variables in the problem correspond to an incident wave $\mathrm{S}$, a reflected wave $\mathrm{R}$, and a transmitted wave $\mathrm{T}$. Each of these three waves has a matrix of $\mathrm{N}$ by $\mathrm{N}$ points representing each of its transverse field components: $\mathrm{E}_{\mathrm{x}}, \mathrm{E}_{\mathrm{y}}, \mathrm{H}_{\mathrm{x}}$, and $\mathrm{H}_{\mathrm{y}}$. Although the magnetic field components are involved in the linear system, it is only required to find the electric field components from which the other components can be found. This means that the linear system will include only electric fields values.

The number of unknowns in this system is the sum of the following:

Twice the number of modes since each mode requires a value for the amplitude of the forward travelling wave and an amplitude for the backward one. 
- $\quad$ Twice the number of grid points for the reflected field since each point has an $E_{x}$ and an $\mathrm{E}_{\mathrm{y}}$ component.

- $\quad$ Also twice the number of grid points for the transmitted field.

With $\mathrm{M}$ modes and $\mathrm{N}$ by $\mathrm{N}$ grid points, the above unknowns add up to $\left(4 \mathrm{~N}^{2}+2 \mathrm{M}\right)$. In order for the linear system to be consistent, the number of equations has to be equal to the number of unknowns. The equations in this case are generated from the continuity of both, the electric and magnetic $\mathrm{x}$ and $\mathrm{y}$ components across the input and output boundaries. So, by matching $\mathrm{E}_{\mathrm{x}}, \mathrm{E}_{\mathrm{y}}, \mathrm{H}_{\mathrm{x}}$, and $\mathrm{H}_{\mathrm{y}}$ at $\mathrm{z}=0$ at every point in the $\mathrm{N}$ by $\mathrm{N}$ grid and again at $\mathrm{z}=\mathrm{w}$, where $\mathrm{w}$ is the thickness of the mask, one finally gets $\left(8 \mathrm{~N}^{2}\right)$ equations altogether.

The technique is based on representing the free space at the input of the mask as a waveguide. Its refractive index profile consists of only ones representing a box filled with air. The same is done for the free space at the output. In this case, the mode solver gives the patterns for the input and output free space waveguide modes. Matching the field components accordingly gives:

E-field at the input: $\left[e_{w g}\right]\left[A_{w g}\right]+\left[e_{w g}\right]\left[B_{w g}\right]=\left[e_{f s_{-} \text {in }}\right]\left[A_{f s_{-} \text {in }}\right]+\left[e_{f s_{-} i n}\right]\left[B_{f s_{-} \text {in }}\right]$

H-field at the input: $\left.{ }^{\left[h_{w g}\right]}\right]\left[A_{w g}\right]-\left[h_{w g}\right]\left[B_{w g}\right]=\left[h_{f s_{-} \text {in }}\right]\left[A_{f s_{-} \text {in }}\right]-\left[h_{f s_{-} i n}\right]\left[B_{f s_{-} \text {in }}\right]$

E-field at the output: $\quad\left[e_{w g}\right]\left[A_{w g}\right]+\left[e_{w g}\right]\left[B_{w g}\right]=\left[e_{f s_{-} \text {out }}\right]\left[A_{f s_{-} \text {out }}\right]$

H-field at the output: $\quad\left[h_{w g} 2\right]\left[A_{w g}\right]-\left[h_{w g}\right]\left[B_{w g}\right]=\left[h_{f s_{-} \text {out }}\right]\left[A_{f s_{-} \text {out }}\right]$

where $\mathrm{e}_{\mathrm{wg} 1}, \mathrm{e}_{\mathrm{wg} 2}$, and $\mathrm{e}_{\mathrm{wg} 3}$ are $2 \mathrm{~N}^{2} \mathrm{x} \mathrm{M}$ matrixes where each column represents a mode with the upper half of the matrix representing the $\mathrm{E}_{\mathrm{x}}$ components of the $\mathrm{N} \times \mathrm{N}$ points of the mode and the lower half of the matrix representing the $E_{y}$ components. $e_{w g 1}$ represents the fields of the forward and the backward modes at the input of the waveguide. $\mathrm{e}_{\mathrm{wg} 2}$ represents the forward travelling mode at the output while $\mathrm{e}_{\mathrm{wg} 3}$ represents the backward travelling mode also at the output.

$\mathrm{e}_{\mathrm{wg} 2}$ and $\mathrm{e}_{\mathrm{wg} 3}$ are related to $\mathrm{e}_{\mathrm{wg} 1}$ through a forward and a backward propagation matrix, respectively. The same applies for the $\mathrm{h}_{\mathrm{wg} 1}, \mathrm{~h}_{\mathrm{wg} 2}$, and $\mathrm{h}_{\mathrm{wg} 3}$ matrices which correspond to the H-field components.

$\mathrm{e}_{\mathrm{fs}_{\_} \text {in }}$ and $\mathrm{e}_{\mathrm{fs} \_ \text {out }}$ are similar to $\mathrm{e}_{\mathrm{wg} 1}, \mathrm{e}_{\mathrm{wg} 2}$, and $\mathrm{e}_{\mathrm{wg} 3}$ but represent E-field components of the input and output waveguides, respectively. Also $h_{\mathrm{fs} \_ \text {in }}$ and $h_{\mathrm{fs}_{-} \text {out }}$ are similar to $\mathrm{h}_{\mathrm{wg} 1}, \mathrm{~h}_{\mathrm{wg} 2}$, and $h_{\mathrm{wg} 3}$ but represent the $\mathrm{H}$-field components of the input and output waveguides, respectively.

$\mathrm{A}_{\mathrm{wg}}, \mathrm{A}_{\mathrm{fs} \_ \text {in }}$, and $\mathrm{A}_{\mathrm{fs} \_ \text {out }}$ are $\mathrm{Mx} 1$ matrices representing the amplitudes of the $\mathrm{M}$ forward propagating modes in the mask waveguide, the input free space waveguide, and the output free space waveguide, respectively. Similarly, $\mathrm{B}_{\mathrm{wg}}, \mathrm{B}_{\mathrm{fs} \_ \text {in }}, \mathrm{B}_{\mathrm{fs}_{-} \text {out }}$ represent the corresponding backward propagating modes. 
In eqns. (1) the matrix $A_{f_{s} \text { in }}$ is chosen to represent a normally incident plane wave (or any other excitation), while $\mathrm{A}_{\mathrm{wg}}, \mathrm{B}_{\mathrm{wg}}, \mathrm{B}_{\mathrm{f} \_ \text {_in }}$, and $\mathrm{A}_{\mathrm{fs} \_ \text {out }}$ are unknowns to be determined. Once $\mathrm{A}_{\mathrm{fs} \_ \text {out }}$ is found, the E-field of the transmitted output of the mask is found as $\left[\mathrm{e}_{\mathrm{fs} \_ \text {out }}\right]\left[\mathrm{A}_{\mathrm{f} \mathrm{f}_{\text {out }}}\right]$.

To reduce the memory requirements, a pseudo inverse operation is used instead of a proper inverse for the matrix. By using the pseudo inverse operation, the number of equations can exceed the number of unknowns converting the problem to a fitting problem. In other words one will get the best solution according to a certain criteria instead of the actual solution. If the number of unknowns is much less than the given equations, even the best solution can be far away from the actual solution. Also if the structure has horizontal and vertical symmetry, the size of the grid can be reduced to one quarter with the appropriate boundary conditions given to the mode solver depending on the polarization of the excitation.

\section{Simulation Results:}

First, we investigate the diffraction effects on a one-dimensional axicon lens as its aperture size L decreases. Ideally, a 1D Bessel beam (which is actually a cosine function 1D representation), results from the superposition of two plane waves intersecting at different angles, where each one of the two plane waves extends from negative infinity to positive infinity. Such an ideal case would result in an infinite depth of field for the resulting beam. Practically, the plane waves must have a finite extension resulting in a finite focal length and finite depth of field. However, if the plane waves extend to a region that is too small, say in the order of a wavelength, the plane wave itself would not behave as a plane wave. Instead, it can be seen as a spherical (cylindrical) wave originating from a point (line) source. In this case, the resulting intensity distribution would deviate from a conventional 1D Bessel beam distribution. In the next section, 1D Bessel beams are generated by super imposing two intersecting plane waves with different directions. A wavelength of $637 \mathrm{~nm}$ was used. The plane waves are represented in the simulations as a set of point sources with unity magnitudes separated by $5 \mathrm{~nm}$ extending over the entire aperture. Figure 2 shows the simulation results for the intensity distribution for apertures ranging from $\mathrm{L}=70 \mu \mathrm{m}$ down to $\mathrm{L}=10 \mu \mathrm{m}$. The phases of the point sources are adjusted to achieve the required angle for each of the two intersecting plane waves. As can be seen, for large apertures $(\mathrm{L}=70 \mu \mathrm{m})$ the diffraction effects are minimal and the pattern represents two plane waves intersecting and resulting in a 1D Bessel beam. As the aperture size decreases, each one of the two plane waves is altered by diffraction effects. The intensity distribution obtained therefore deviates from the usual Bessel beams. Both, the focal length and the depth of field are reduced as seen in Figure 2. For $\mathrm{L}=20 \mu \mathrm{m}$, one can still recognize the two intersecting plane waves, whereas for $\mathrm{L}=10 \mu \mathrm{m}$, the distribution is closer to that of a single slit. In 
Figures $3 \mathrm{a}$ through $3 \mathrm{e}$, the intensity is plotted along the $\mathrm{z}$-direction for the same apertures.

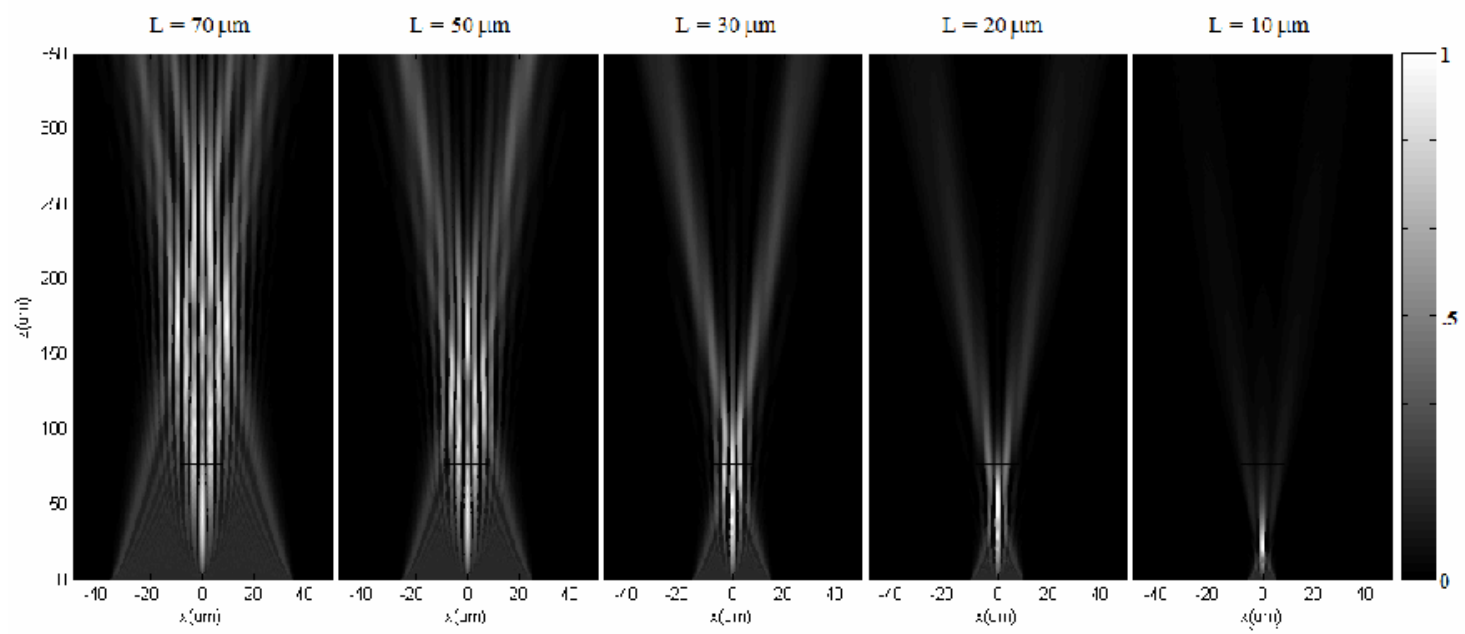

Figure (2): Intensity distribution $|E(x, z)| 2$ for different aperture ranging from $L=70 \mu \mathrm{m}$ down to $L=10 \mu \mathrm{m}$.

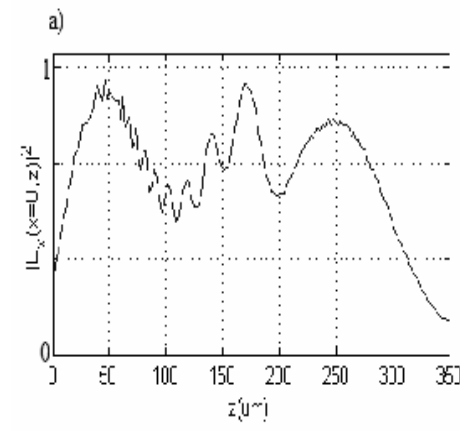

d)

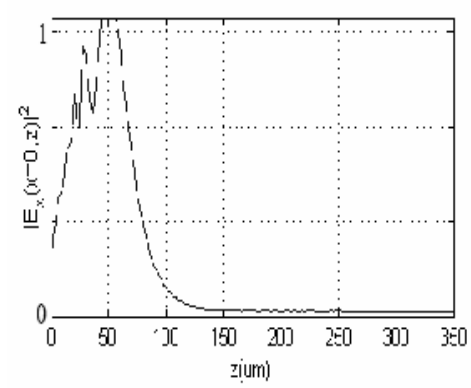

b)

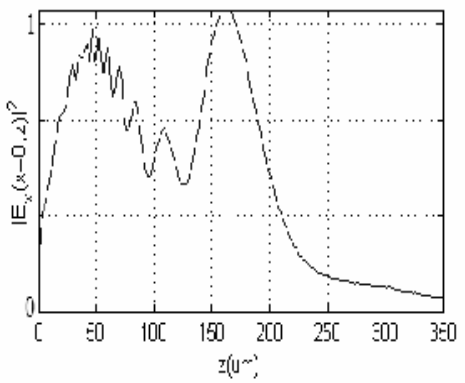

ह)

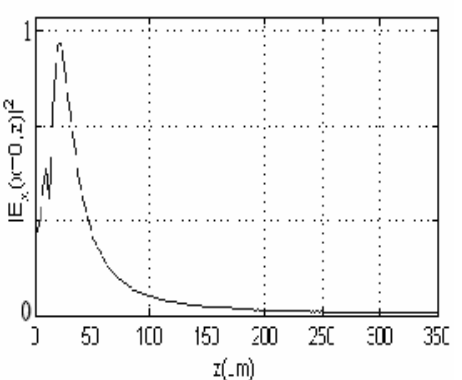

Figure (3): Intensity plotted along the z-direction for the different apertures: a) $L=70 \mu \mathrm{m}, b) L=50 \mu \mathrm{m}, \mathrm{c}) \mathrm{L}=30 \mu \mathrm{m}, d) \mathrm{L}=20 \mu \mathrm{m}$, and e) $L=10 \mu \mathrm{m}$.

The results shown in Figure 2 and 3 are for ideal point sources as stated previously. In the next section, the sources are replaced by the output from the proposed planar lens. The lens consists of a set of 33 slits. The phase of the output from a slit is controlled by 
changing the width of the slit. The structure shown in Figure 1 has only 9 slits just for illustration. The widths of the 33 slits range from $30 \mathrm{~nm}$ at the centre up to $130 \mathrm{~nm}$ at the sides. The excitation was taken as a normally incident plane wave polarized perpendicular to the slit edges which is represented by PW1 in Figure 1. With a wavelength of $637 \mathrm{~nm}$, the spacing between the slits was swept from $300 \mathrm{~nm}$ up to $600 \mathrm{~nm}$ to control the angles of the output plane waves PW2 and PW3 as shown in Figure 1 and consequently control the depth of field of the resulting Bessel beam. The thickness of the conducting slab was taken as $500 \mathrm{~nm}$ with relative permittivity of $17.36+\mathrm{j} 0.715$ and assuming air on both sides of the slab as stated previously.

The structure is simulated using the modal technique explained in part 3. A window size of 3000 mesh points with a step size of $10 \mathrm{~nm}$ was chosen. The number of modes was taken as 100. The output from the modal technique presenting the $\mathrm{x}$ and $\mathrm{y}$ field components at the 3000 mesh points at the output of the slab is then propagated in free space by using Fourier optics technique. This is based on taking the field Fourier transform then propagating each spatial component in free space by using its corresponding propagation constant. After this phase shifting, the field is returned to the spatial domain by using again the inverse Fourier transforms to get the field patterns. Examples of the intensity distribution along the z-axis, are shown in Figure 4.

In Figure 4, the intensity distribution $|\mathrm{E}(\mathrm{x}, \mathrm{z})|^{2}$ is shown for different values of the spacing $S$ between the apertures as $S=300 \mathrm{~nm}, 400 \mathrm{~nm}, 500 \mathrm{~nm}$, and $600 \mathrm{~nm}$. As can be seen, the focal length (or depth of focus) (DOF) increases with increasing S. In Figure 5, the intensity is plotted along the $\mathrm{Z}$-direction for the same values for the spacing $\mathrm{S}$.

The spacing between the apertures is extended further to 1000nm in Figure 6 and Figure 7 where the focal length and the depth of field are plotted versus spacing, respectively. 

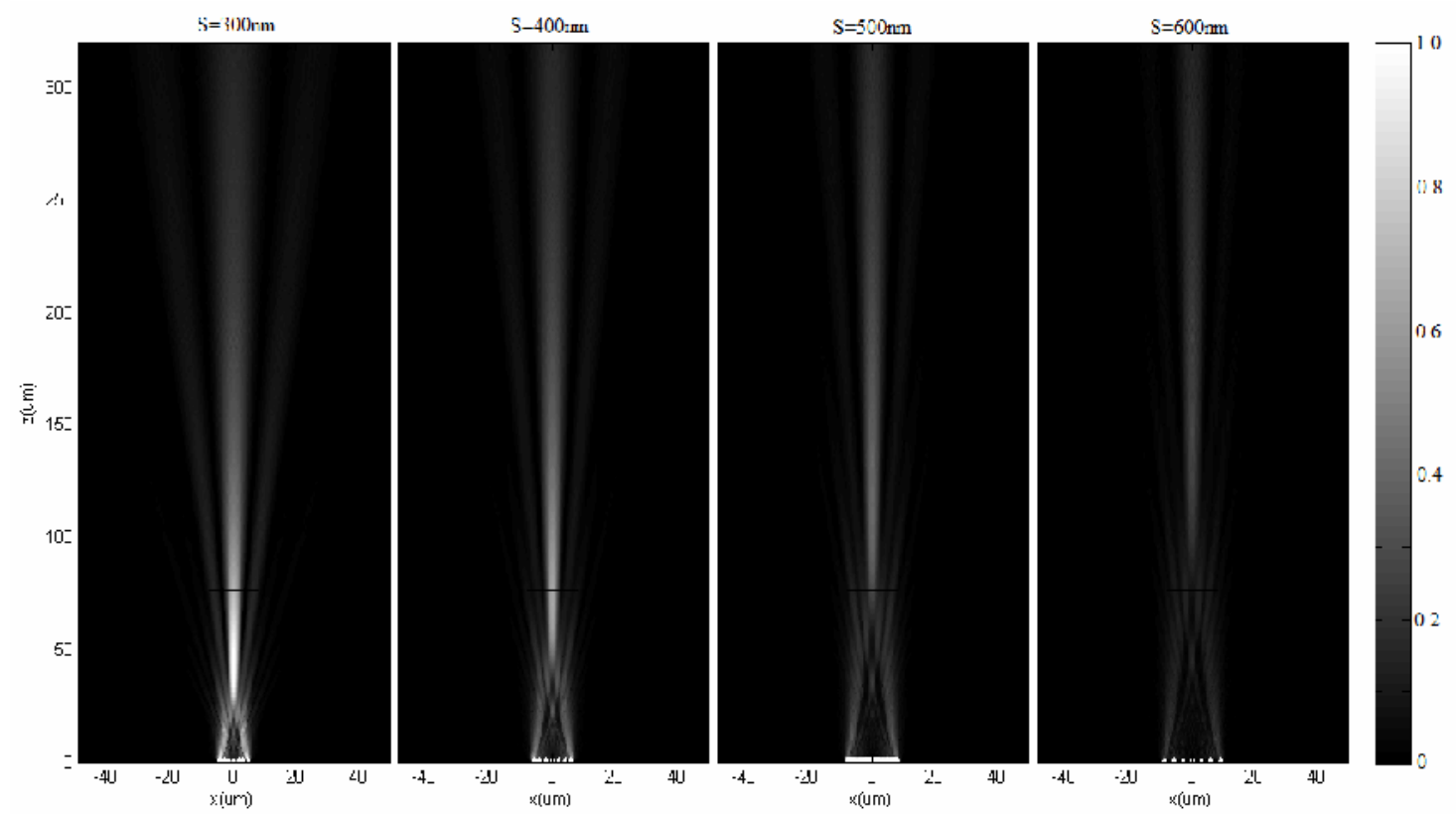

Figure (4): Intensity distribution $|E(x, z)|^{2}$ obtained from actual slits of different spacing $S$ between the slits. The spacing $S$ between the slits ranges from $S=300 \mathrm{~nm}$ to $S=600 \mathrm{~nm}$.
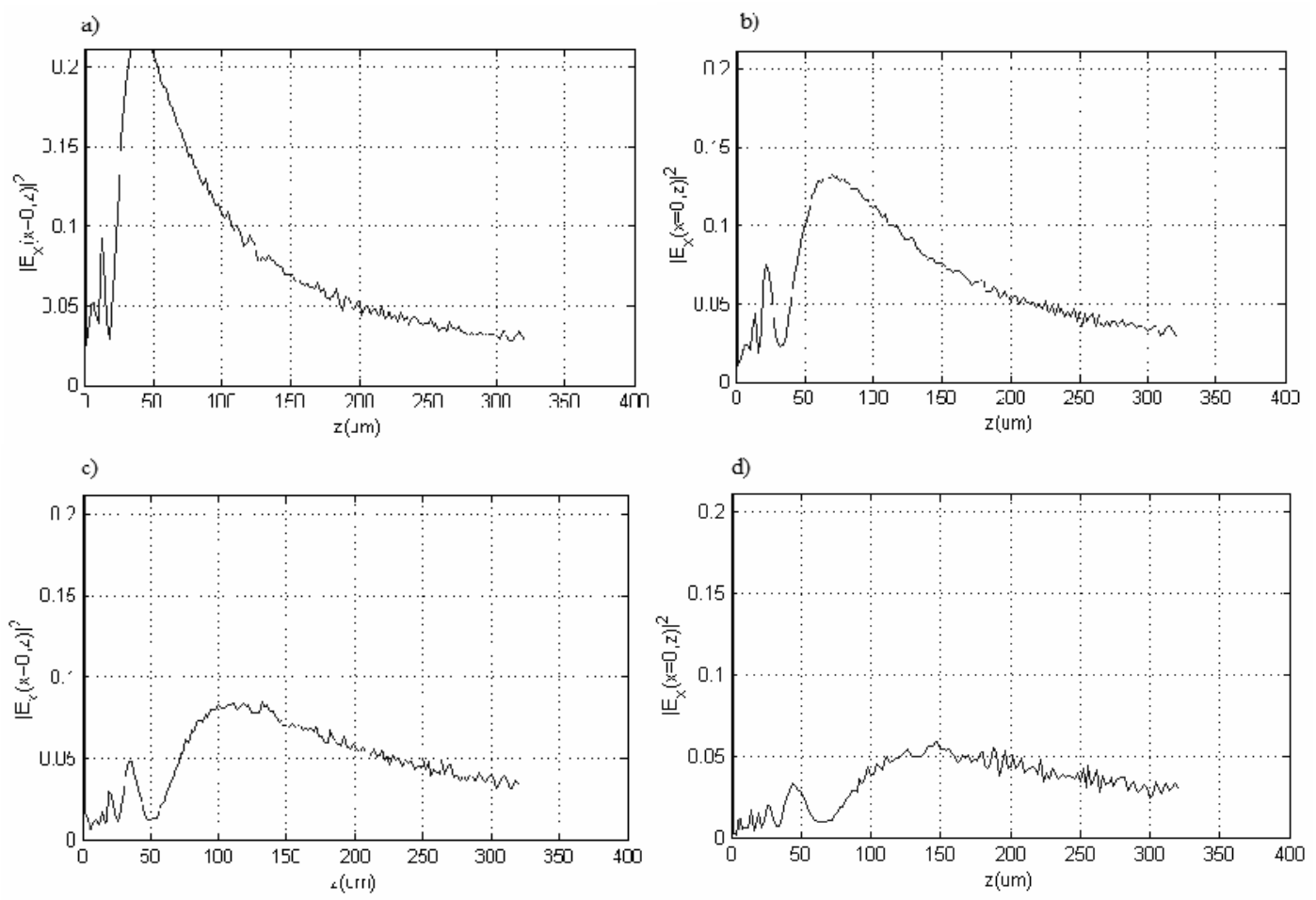

Figure (5): Intensity plotted along the z-direction for different slit separation S: a) $S=300 \mathrm{~nm}, b) S=400 \mathrm{~nm}, \mathrm{c}) \mathrm{S}=500 \mathrm{~nm}$, and d) $S=600 \mathrm{~nm}$. 


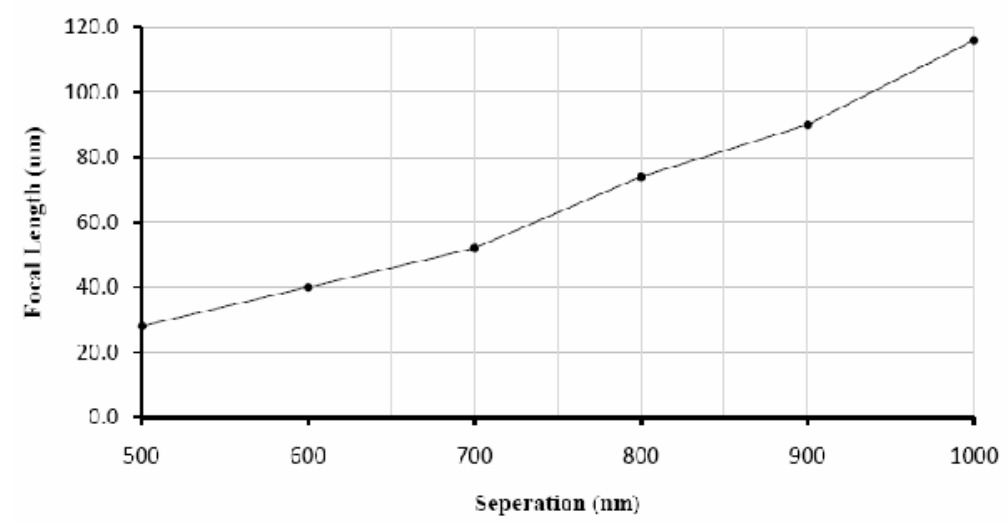

Figure (6): Dependence of the focal length on the slit separation.

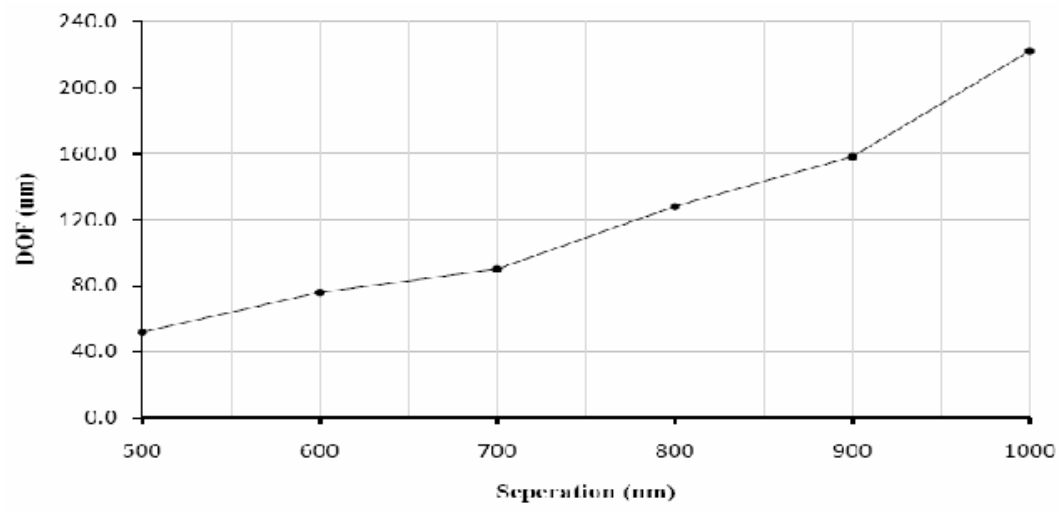

Figure (7): Dependence of the depth of field on the slit separation.

\section{Discussion and Conclusions:}

The above simulations show that reducing the overall lens size causes the characteristics of the lens to deviate from the ideal Bessel beam and as the size is reduced further to nanoscale dimensions, the diffraction affects the behaviour significantly so that the obtained beam can no longer be considered a low diffraction Bessel beam. However, for an overall lens size in the order of $30 \mathrm{um}$ or even $20 \mathrm{um}$, a reasonable DOF can still be achieved. This fact was used for constructing the proposed nanoscale planar lens and controlling its focal length and DOF by changing the spacing between the apertures. Figure 6 and Figure 7 show that with aperture spacing ranging from 500nm to $1000 \mathrm{~nm}$ corresponding to an overall lens size between approximately $17 \mu \mathrm{m}$ and $34 \mu \mathrm{m}$ we can achieve focal lengths ranging from around $30 \mu \mathrm{m}$ to $120 \mu \mathrm{m}$ with a DOF of around $50 \mu \mathrm{m}$ and $220 \mu \mathrm{m}$, respectively. It is therefore concluded that nanoscale planar lenses are very promising especially for the design of axicon like lenses where a large focal length and DOF are required. 


\section{References:}

[1] L. Verslegers, P. B. Catrysse, Z. Yu, J. S. White, E. S. Barnard, M. L. Brongersma, and S. Fan, "Planar lenses based on nanoscale slit arrays in a metallic film," Nano Lett. 9(1), 235-238, 2009.

[2] Osman S. Ahmed, Mohamed. A. Swillam, Mohamed. H. Bakr, and Xun Li, "Modeling and design of nano-plasmonic structures using transmission line modeling," Optics Express, vol 18, no. 21 (2010).

[3] Haofei Shi, Changtao Wang, Chunlei Du, Xiangang Luo, Xiaochun Dong, Hongtao Gao, "Beam manipulating by metallic nano-slits with variant widths," Optics Express, vol 15, no. 18 (2005).

[4] A. Taflove and S. C. Hagness, Computational Electrodynamics: the FiniteDifference Time-Domain Method, Artech House, Boston, Mass., 2000

[5] Jaione Tirapu Azpiroza, Geoffrey W. Burrb, Alan E. Rosenbluthc, and Michael Hibbs, "Massively-parallel FDTD simulations to address mask electromagnetic effects in hyper-NA immersion lithography," Optical Microlithography, vol. 6924, 2008

[6] Arman B. Fallahkhair, and Thomas E. Murphy, "Vector finite difference modesolver for anisotropic dielectric waveguides," J. Lightw. Technol., vol. 26, no. 11 , pp. $1423-1431,2008$. 